\title{
CHARACTERIZATION OF UNIVARIATE LONG-TERM URBAN INTERNET TRAFFIC VOLUME
}

\author{
Subhasish Debroy ${ }^{1}$, Rajdeep Ray ${ }^{2}$, Mofazzal Hossain Khondekar ${ }^{3}$, Baisakhi Chakraborty ${ }^{4}$ and \\ Anup Kumar Bhattacharjee ${ }^{5}$
}

\author{
${ }^{1}$ Department of Computer Applications, Dr. B.C. Roy Engineering College, India \\ ${ }^{2}$ Department of Electronics and Communication Engineering, Dr. B. C. Roy Engineering College, India \\ ${ }^{3}$ Department of Applied Electronics and Instrumentation Engineering, Dr. B. C. Roy Engineering College, India \\ ${ }^{4}$ Department of Computer Science and Engineering, National Institute of Technology, Durgapur, India \\ ${ }^{5}$ Department of Electronics and Communication Engineering, National Institute of Technology, Durgapur, India
}

\begin{abstract}
The proposed work deals with a real time hourly internet traffic data set in bits collected from ISPs located in 11 cities of European Country for the period $7^{\text {th }}$ June 2005 to $31^{\text {st }}$ July 2005. Then a thorough statistical inference has been drawn regarding the central tendency, dispersion and distribution of the data. Time-frequency analysis using Smoothed Pseudo Wigner Ville Distribution (SPWVD) is implied to infer knowledge about the non-stationarity of the system. A nonparametric test for normality, Anderson Darling Test (AD-Test) has been performed to detect the binary signature of nonlinearity in the signal. Delay Vector Variance Analysis $(D V V)$ are being exploited to infer deeper knowledge about the determinism and nonlinearity in the system. The results confirm a nonstationary, relatively stochastic and nonlinear profile of the signal under observation.
\end{abstract}

Keywords:

Time-Frequency Analysis, Smoothed Pseudo Wigner Ville Distribution SPWVD, Anderson Darling Test (AD-Test), Delay Vector Variance Analysis (DVV), Nonlinearity

\section{INTRODUCTION}

The Internet traffic is growing up and being scattered over the world day by day. The increased traffic rate introduces so many difficulties regarding bandwidth, speed, buffering, streaming congestion etc. which in turn increases the dynamical complexity of the network. Internet traffic depends on flow of data across the Internet. The volume of data usage, transfer and storage has become alarming in present days. The Service providers are under huge challenge due to continuous high demand for low cost multiple services through internet. This results in a requirement of a rigorous and continuous real time assessment of the technological and infrastructural up-gradation of network systems to achieve sufficient quality of service (QOS) [1]. Without proper interpretation and treatment of the internet data traffic flow it is difficult to cope with such a bottle neck. The proper interpretation of the Internet Traffic is to understand the traffic characteristics, its prediction and performance analysis. Due to increasing number of nodes, usage of internet and popularization of network technology traffic being increased, in such cases various research works have been done to manage the network traffic by some researchers such as Packet sniffers [2]. Some techniques of analyzing network traffic using statistical signal processing depending upon three major issues i.e. Network control, detection of the periodic behavior in network traffic and control in communication channels can be found in some literatures [3] [4]. To manage the network traffic many researchers has given their attention and paying their effort to establish an efficient model [5] [6]. To improve the quality of network system it is so important to analyze the dynamics of the system using real data sets.

The present work primarily deals with the proper processing of the data to be analyzed. Then a thorough statistical inference has been drawn regarding the central tendency, dispersion and distribution of the data using summary statistics and probability density function (pdf). Time-frequency analysis is implied to infer knowledge about the non-stationarity of the system. Finally Anderson Darling Test (AD-Test) and Delay Vector Variance Analysis (DVV) are being exploited to examine the signature of nonlinearity in the system.

\section{EXPERIMENTAL DATA AND DATA PREPROCESSING}

A real time hourly data set in bits are collected from data market [7] of the ISP located in 11 cities of European Country for the period $7^{\text {th }}$ June 2005 to $31^{\text {st }}$ July 2005 is the data under observation. For the purpose of data preprocessing the trend and the seasonality have been analyzed.

\subsection{TREND ANALYSIS}

The Fig. 1 shows the plot of linear trend of the data. The linear trend line shows a slight monotonically decreasing trend which is negligible to be considered.

\subsection{TEST FOR SEASONALITY}

The Fig. 2 shows the plot of daily seasonal index of the data. The plot clearly shows presence of weekly seasonality.

Since the data is weekly seasonal and the trend is negligible as a customary the data is to be smoothed properly. Here simple exponential smoothing technique is being exploited for the purpose. 


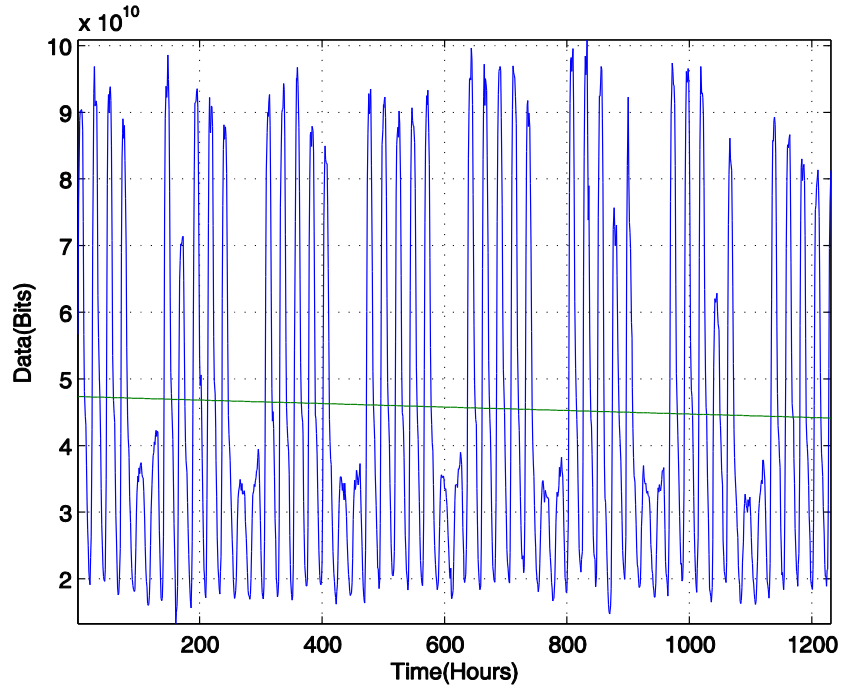

Fig.1. Linear trend estimation

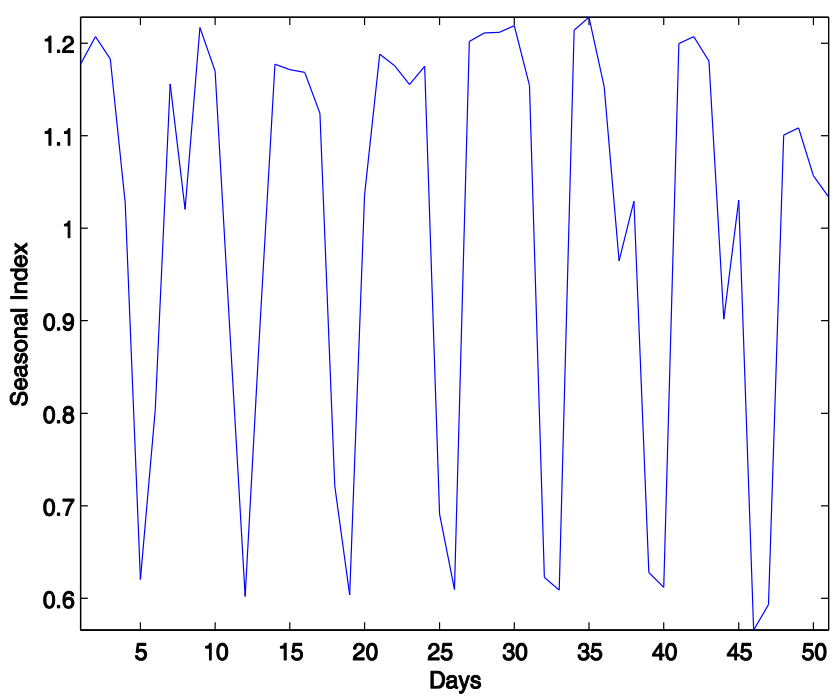

Fig.2. plot of Daily Seasonal index

\subsection{SIMPLE EXPONENTIAL SMOOTHING}

Exponential Smoothing [8] [9] assigns exponentially decreasing weights as the observation get older. In other words, recent observations are given relatively more weight in forecasting than the older observations. If the original data series be $\left\{X_{i}\right\} ; i=1,2,3, \ldots, n$, then the $i^{\text {th }}$ smoothed data $\left\{S_{i}\right\}$ is given by,

$$
\begin{gathered}
S_{i}=\alpha X_{i}+(1-\alpha) S_{i-1} ; \quad i=1,2,3, \ldots, n \\
S_{1}=X_{1}
\end{gathered}
$$

Here, $\alpha$ is smoothing constant which lies between $(0<\alpha<1)$ and considered as $\alpha=0.5$ in this work. The Fig. 3 shows the plots of original data and smoothed data. So before testing the linearity of the time series data, it is more necessary to test the stationary of this time series data.
Table.1. Statistical summary of Smoothed Data

\begin{tabular}{|c|c|}
\hline Scores & Smoothed Data (bits) \\
\hline Mean & 45708148212.0866 \\
\hline Median & 35931547779.6733 \\
\hline Mode & 56718587433.0000 \\
\hline Standard Deviation & 24222575798.0282 \\
\hline Variance & $5.86733178291222 \times 10^{20}$ \\
\hline Maximum & 97979546424.7370 \\
\hline Minimum & 15742053181.8430 \\
\hline Skewness & 0.688412281925276 \\
\hline Kurtosis & 2.07253090704951 \\
\hline
\end{tabular}
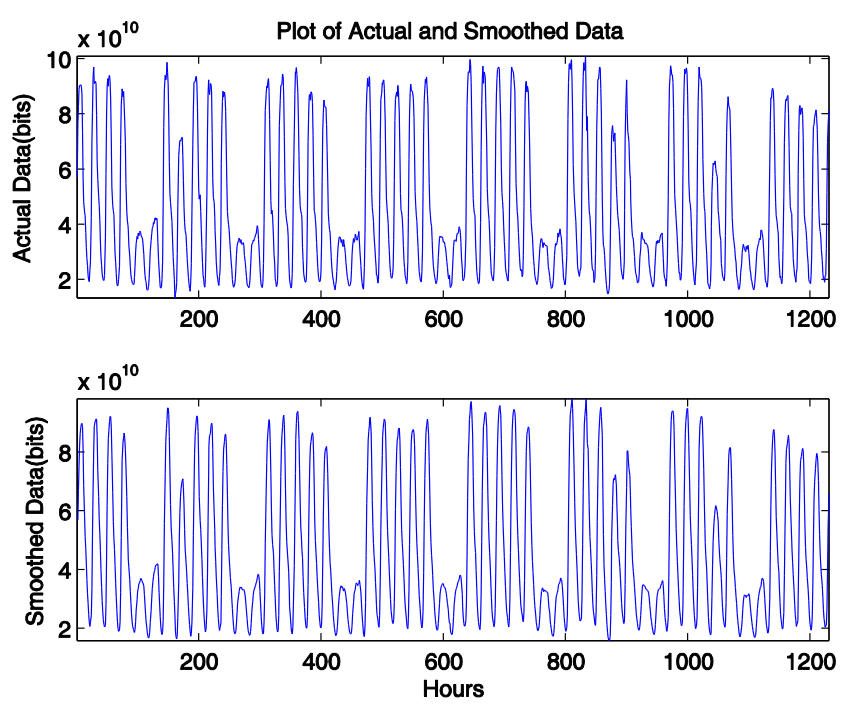

Fig.3. Plots of Actual and Smoothed Data
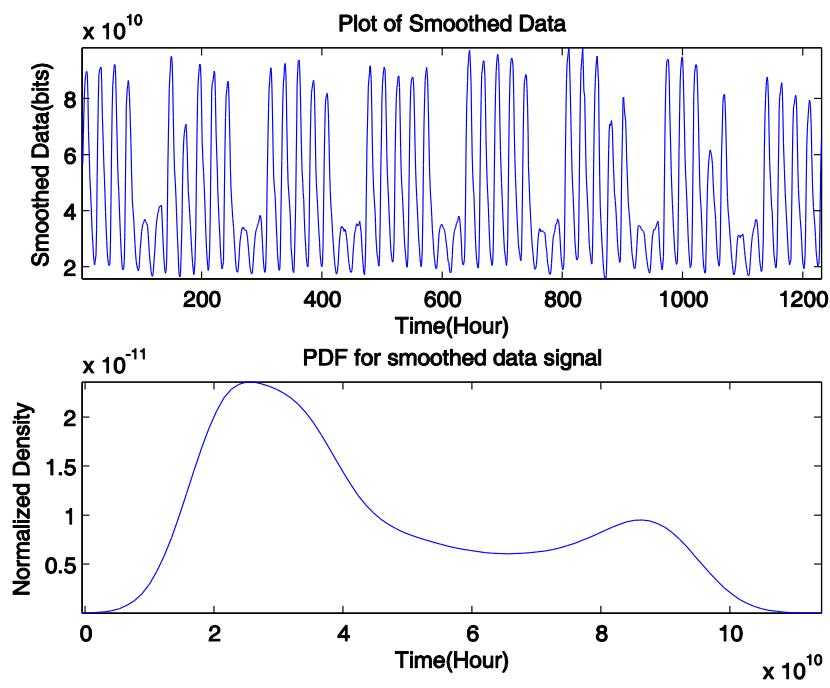

Fig.4. Plot of smoothed data and normalized pdf

The Fig.4 shows the plot of smoothed data and its normalized probability density function (pdf). The summary statistics of the experimental data are tabulated in Table.1. The Table.1 basically exhibits a statistical report under the investigation of the real time 
original data and smoothed data signal. The pdf shows two unequal peaks or local maxima which signifies that the distribution is bimodal [10]. The larger mode is called major mode or acrophase and the fewer mode is called minor mode and the least frequent value between these two modes is known as antimode or batiphase. Generally bimodal distribution is the combination of two unimodal distributions. Here the major mode is due to the working days of a week and the minor mode is due to the week-end low consumption of data.

\section{TIME FREQUENCY ANALYSIS}

As real life signals change over time, transitional or nonstationary components are an inevitable part of these signals. The components can be hardly analyzed based on only the time or the frequency, and therefore time-frequency tools were introduced.

\subsection{SMOOTHED PSEUDO WIGNER VILLE DISTRIBUTION (SPWVD)}

The Wigner-Ville Distribution (WVD) is one of the basic time-frequency distribution (TFD) with optimal resolution. In addition, the WVD exhibits many beneficial mathematical properties, including energy conservation, time and frequency shift invariants, or compatibility with filters; the details are described by [11]. For the removal of the cross terms formed in the frequency plane, windowing in time is needed. In consequence of this fact, smoothing in time and frequency was introduced. The derived Smoothed Pseudo Wigner-Ville Distribution [12]-[15] enables the reduction of all types of interferences at the cost of resolution reduction [16].

The approach is based on the use of the autocorrelation function for calculating the power spectrum. To construct the autocorrelation function, the signal is compared to itself for all possible relative shifts, or lags. This is equivalent to a frequency smoothing of the WVD. The Wigner-Ville (and all of Cohen's class of distribution) uses a variation of the autocorrelation function where time remains in the result, called instantaneous autocorrelation function.

The Wigner Distribution is defined as;

$$
W_{X}(t, f)=\int_{-\infty}^{\infty} Z(t+\tau / 2) Z^{*}(t-\tau / 2) e^{-j 2 \pi f \tau} d \tau
$$

where, $Z$ is the analytic signal. by

From the viewpoint of spectrum the WVD can be described

$$
W_{X}(t, f)=\int_{-\infty}^{\infty} Z(f+\xi / 2) Z^{*}(\omega-\xi / 2) e^{-j 2 \pi \xi \tau} d \xi
$$

where, $*$ denotes the complex conjugate.

Now for continuous signal the SPWVD is given by

$$
\begin{aligned}
& \operatorname{SPWVD}(t, v) \\
& =\int h(\tau)\left[\int g(s-t) x\left(s+\frac{\tau}{2}\right) x^{*}\left(s-\frac{\tau}{2}\right) d s\right] e^{-j 2 \pi v \tau} d \tau
\end{aligned}
$$

where, $h(\tau)$ denotes frequency smoothing of interference oscillating in parallel to the frequency axis and $g(t)$ denotes temporal smoothing of interference oscillating in parallel to the temporal axis. The kernel function to filter out the interferences independently is given by,

$$
f(\xi, \tau)=e^{-\left(\frac{\Pi \xi}{\sqrt{2} \sigma_{\xi}}\right)^{2}} e^{-\left(\frac{\Pi \tau}{\sqrt{2} \sigma_{\tau}}\right)^{2}}
$$

Using Eq.(5) and Eq.(6) the expression for SPWVD becomes,

$$
\operatorname{SPWVD}(t, v)=\sqrt{\frac{2}{\pi}} \int e^{-\left(\frac{\pi \tau}{\sqrt{2} \sigma_{\tau}}\right)^{2}} e^{-j 2 \pi v \tau} \beta d \tau
$$

where,

$$
\beta=\left[\int \sigma_{\xi} \xi e^{-2 \sigma^{\xi^{2(t-s)^{2}}}} x\left(s+\frac{\tau}{2}\right) x^{*}\left(s-\frac{\tau}{2}\right) d s\right]
$$

The SPWVD for discrete signal is given by,

$$
\begin{aligned}
& \operatorname{SPWVD}(n, m)=2 \sqrt{\frac{2}{\pi}} \sum_{k=-h}^{h} e^{-\frac{2 \pi k}{\sigma_{\tau} \sqrt{2}}} \\
& \sum_{u=-g}^{g} \sigma_{\xi} e^{-2\left(\sigma_{\xi} u\right)^{2}} x(n+u+k) x(n+u-k) e^{-j 4 \pi m k}
\end{aligned}
$$

\section{DETECTING DETERMINISM AND NON- LINEARITY IN THE PROCESS}

The detection of nonlinearity and determinism in time series is one of the most intense research fields in nonlinear time series analysis. Due to the difficulty in obtaining reliable and clear-cut results from time series of natural phenomena, different methods have to be used in combination to assess the coherence of the results obtained. Several methods are there to test the determinism and linearity of the time series data. Here Anderson Darling Test (AD-Test) and DVV Analysis are being exploited for the purpose.

\subsection{ANDERSON DARLING TEST (AD-TEST)}

Anderson-darling Test [17] is a statistical analysis for distribution type. The fundamental principle of this AD-Test is that if the data is not normally distributed, then it is considered as nonlinear. If $Y_{i}$ is considered as the original data and $F$ is the Cumulative Distribution Function, then a test statistic $A^{2}$ can be given by,

$$
A^{2}=-n-\sum_{i=1}^{n}\left(\frac{1}{n}\right)\left(2_{i}-1\right)\left[\ln \left(F\left(Y_{i}\right)\right)+\ln \left(1-F\left(Y_{n+1-i}\right)\right)\right]
$$

where, $i$ is the number of iteration i.e. $i=1,2,3, \ldots, n$ and $n$ is the sample size.

For a significance level of $99 \%$ the critical value of $A^{2}$ is 0.787 . A value of $A^{2}>1.092$ will specify the system dynamics as nonlinear.

\subsection{DELAY VECTOR VARIANCE ANALYSIS (DVV)}

Delay Vector Variance (DVV) algorithm [18] [19] determines the presence of non-linear and stochastic nature of time series both in the presence and absence of chaos. This method requires the underlined time series to be represented in phase space with 
optimum embedding dimension and time lag. There are basically three phases of DVV.

\subsubsection{Phase I: Time Delay Embedding:}

In a single time series the time can be embedded using embedding dimension $m$ and the time lag $\tau$. A single component of the embedded time is known as delay vector (DV). Hence the phase space of the time series consists of a group of delay vectors (DVs).

Step 1: If $X$ be the variable of the time series then a DV can be expressed as,

$$
x(k)=\left\{X_{k-m \tau}, \ldots, X_{k-\tau}\right\}^{T}
$$

Every DV has its corresponding target or the next sample $x_{k}$. In this step all $x(k)$ and $x_{k}$ are estimated.

\subsubsection{Phase II: Characterization of Time Series:}

Step 2: All the pair wise Euclidean distance between DVs, $d(i, j)$ $=\|x(i)-x(j)\|,(i \neq j)$ are computed then.

Step 3: The DVs which are within a certain Euclidean distance to DV $x(k)$ are grouped into $\lambda_{k}$ sets. This Euclidean distance will be varied in a manner standardized with respect to the distribution of pair wise distances between DVs. The minimum acceptable number of DVs in a single set is 30 .

Step 4: Now the mean $\mu_{d}$ and the standard deviation $\sigma_{d}$ are computed over all pair wise Euclidean distance between DVs.

Step 5: The sets $\lambda_{k}\left(r_{d}\right)$ are generated such as $\lambda_{k}\left(r_{d}\right)=\{x(k)\}$ $\|x(k)-x(i)\| \leq r_{d}$, i.e. sets which consists of all DVs that lie closer to $x(k)$ than a certain distance $r_{d}$, taken from the interval $\left[\min \left\{0, \mu_{d}-\eta_{d}\right\} ; \mu_{d}+\eta_{d} \sigma_{d}\right]$, where $\eta_{d}$ is a parameter controlling the span over which to perform DVV analysis.

Step 6: For every $\operatorname{set} \lambda_{k}\left(r_{d}\right)$, the variance of the corresponding targets $\sigma_{k}^{2}\left(r_{d}\right)$ is computed. The average over all sets $\lambda_{k}\left(r_{d}\right)$, normalized by the variance of the time series signal, $\sigma_{k}^{2}$, yields the 'target variance'

$$
\sigma^{* 2}\left(r_{d}\right)=\frac{\frac{1}{N} \sum_{k=1}^{N} \sigma_{k}^{2}\left(r_{d}\right)}{\sigma_{x}^{2}}
$$

where, $N$ is the total number of sets $\lambda_{k}\left(r_{d}\right)$. For standardization of the distance axis $r_{d}$ is replaced by $\left(r_{d}\right.$ $\left.\mu_{\mathrm{d}}\right) / \sigma_{d}$ having zero mean and unit variance.

Step 7: The minimum target variance $\sigma_{\min }^{* 2}=\min _{r_{d}}\left[\sigma^{* 2}\left(r_{d}\right)\right]$ is then computed.

\subsubsection{Phase III: DVV Plot and DVV Scatter Diagram:}

Step 8: To produce DVV scatter diagram 99 surrogate time series have been generated using Iterative Amplitude Adjusted Fourier Transform (iAAFT) [20]

Step 9: Now the DVV plot is obtained plotting the target variance $\sigma_{k}^{2}\left(r_{d}\right)$ against the standardized distance $\left(r_{d}\right.$ $\left.\mu_{\mathrm{d}}\right) / \sigma_{d}$ for both original and surrogate series.
Step 10: Now plotting the $\sigma_{k}^{2}\left(r_{d}\right)$ of the original time series toward horizontal axis and that of the surrogates toward vertical axis the scatter diagram is produced.

\section{RESULTS AND DISCUSSION}

The Wigner-Ville distribution is the Fourier transform of the signal's autocorrelation function with respect to the delay variable. It can also be thought of as a short-time Fourier transform where the windowing function is a time-scaled, timereversed copy of the original signal. It is a popular tool for timefrequency analysis because generally it has better resolution than the short-time Fourier transform. Calculation of power spectra is based on correlation-like terms, which implicitly considers the temporal relationship of the data. This makes the assumption of data stationary unnecessary [21]. The main advantage of the Wigner-Ville over global spectral analysis is the dynamic representation of the signal structure, both time and frequency characteristics are simultaneously available and the temporal and frequency windows can be adapted to reduce to a minimum the interferences between the different spectral components and to obtain maximal time and frequency resolutions. The timefrequency energy distributions will be in general quadratic representations. SPWVD is obtained by comparing the information of a windowed or a shifted version of the same signal at other times and frequencies. The Fig.5 shows the Timefrequency energy distributions of the signal using SPWVD.

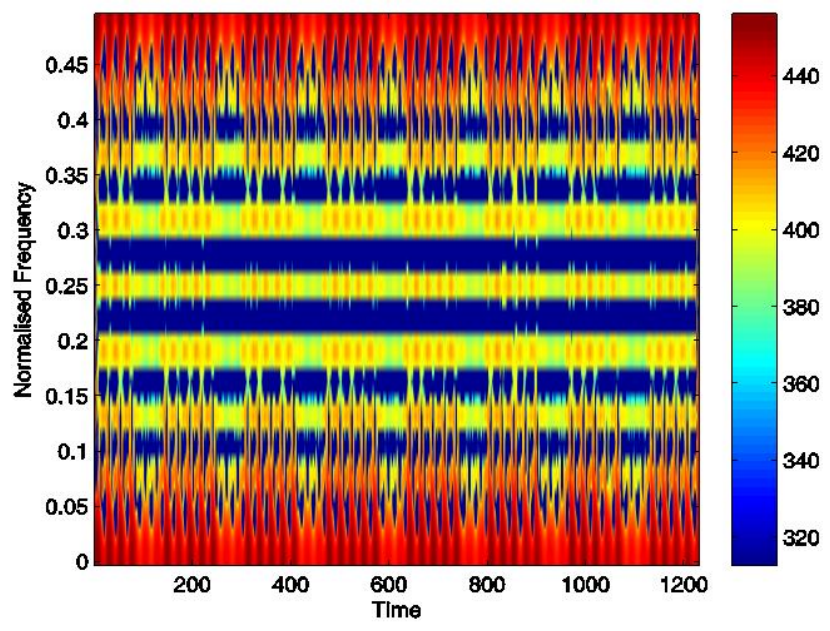

Fig.5. Time-frequency energy distributions of the signal using SPWVD

From Fig.5, it is clear that frequency as well as energy density vary with time for the signal. The presence of different instantaneous frequencies which characterize a local frequency behavior of the signal as a function of time is evident from the inspection. The presence of group delays which is the local time behavior as a function of frequency is also observed. The observations strongly recommends the time series data as Nonstationary.

There are many non-parametric and robust techniques that do not make strong distributional assumptions. However, techniques based on specific distributional assumptions are in general more powerful than non-parametric and robust techniques. Therefore, 
if the distributional assumptions can be validated, they are generally preferred. The probability plot of the data is given in the Fig.6. The Fig.6 shows a clear deviation of the data from the linear normal plot implying the non-normal distribution of the data.

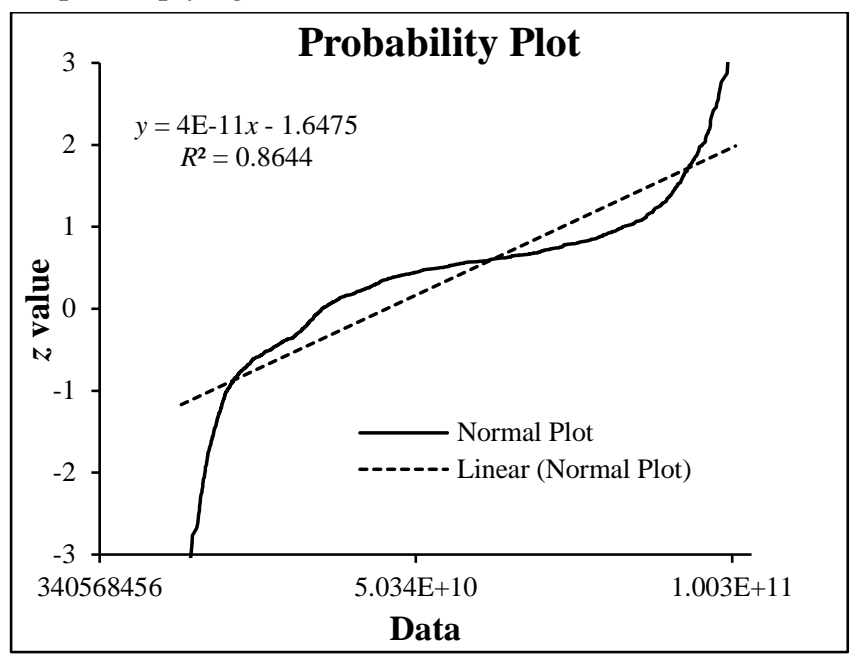

Fig.6. Probability plot of the data

In $\mathrm{AD}$-Test for a significance level of $99 \%$ the value of $A^{2}$ is obtained as 62.505. A value of $A^{2}>1.092$ will reject the hypothesis that the signal came from a normal distribution. The obtained value unambiguously rejects the null hypothesis and hence specify the system dynamics as nonlinear. Using DVV analysis both determinism and nonlinearity can be detected within the signal under observation. For this purpose the embedding dimension $(m)$ and embedding lag $(\tau)$ are to be optimized. This optimization ensures best predictability as well as lowest target variance $\sigma_{\min }^{* 2}$. The lowest target variance $\sigma_{\min }^{* 2}$ is in turn an inverse relative measure of predictability and closely related to the prevalence of the deterministic component over the stochastic one in the data. In order to produce the DVV plot and DVV scatter diagram 99 surrogates [22] have been generated using Iterative Amplitude Adjusted Fourier Transform (iAAFT). The Fig.7 and Fig. 8 shows the DVV plot of original and surrogate data series and DVV scatter diagram respectively. The optimized embedding dimension $(m)$ and embedding lag $(\tau)$ has been computed using differential entropy method [23][24] whereas the lowest target variance $\sigma_{\text {min }}^{* 2}$ estimated from Fig.7 and are tabulated in Table.2. From Table.2, it is evident that as the minimal target variance $\sigma_{\min }^{* 2}$ has a fairly large value of 0.2434 , the signal is relatively more stochastic in nature.

Table.2. Embedding dimension (m), embedding lag ( $\tau$ ), RMSE and $\sigma_{\min }^{* 2}$ for the signal

\begin{tabular}{|c|c|c|c|}
\hline $\begin{array}{c}\text { Embedding } \\
\text { dimension }(\boldsymbol{m})\end{array}$ & $\begin{array}{c}\text { Embedding } \\
\text { lag }(\boldsymbol{\tau})\end{array}$ & RMSE & $\boldsymbol{\sigma}_{\text {min }}^{* 2}$ \\
\hline 5 & 4 & 0.0678 & 0.2434 \\
\hline
\end{tabular}

With the help of original and surrogate time series DVV scatter diagram can be produced, where the horizontal axis corresponds to the DVV plot of the original time series and the vertical to that of surrogate time series. If the surrogate time series signal yields DVV plots similar to the original time series signal, the DVV scatter diagram coincides with the bisector line, then the original time series is judged to be linear and if the time series signal yields DVV plots deviating from the bisector line then the original time series signal judged to be non-linear. This deviation from the bisector line can be quantified as root mean square error (RMSE) between $\sigma^{* 2} s$ of the original time series and $\sigma^{* 2} s$ of the averaged over the DVV plots of the surrogate time series:

$$
t^{D V V}=\sqrt{\sigma^{* 2}\left(r_{d}\right)-\frac{\sum_{i=1}^{N_{s}} \sigma_{s, i}^{* 2}\left(r_{d}\right)}{N_{s}}}
$$

where, $\sigma_{s, i}^{* 2}\left(r_{d}\right)$ is the target variance at span $r_{d}$ for the $i^{\text {th }}$ surrogate and the average is taken over all span of $r_{d}$ that is valid in all surrogate and DVV plots. The root mean square error (RMSE) between $\sigma^{* 2} s$ of the original time series and $\sigma^{* 2} s$ of the averaged over the DVV plots of the surrogate time series has been estimated from Fig. 8 and tabulated in Table.2.

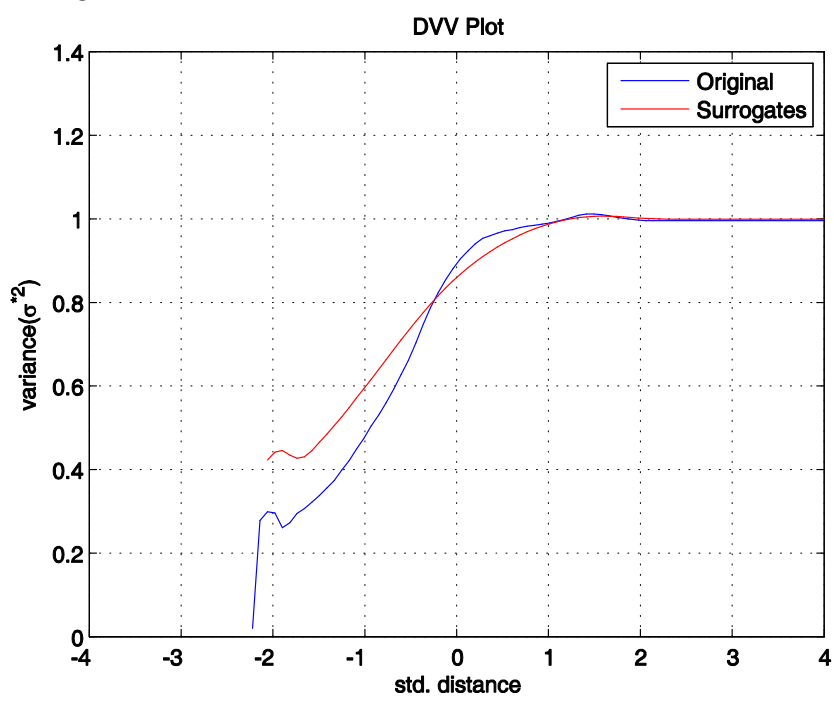

Fig.7. DVV plot of original and surrogate data series

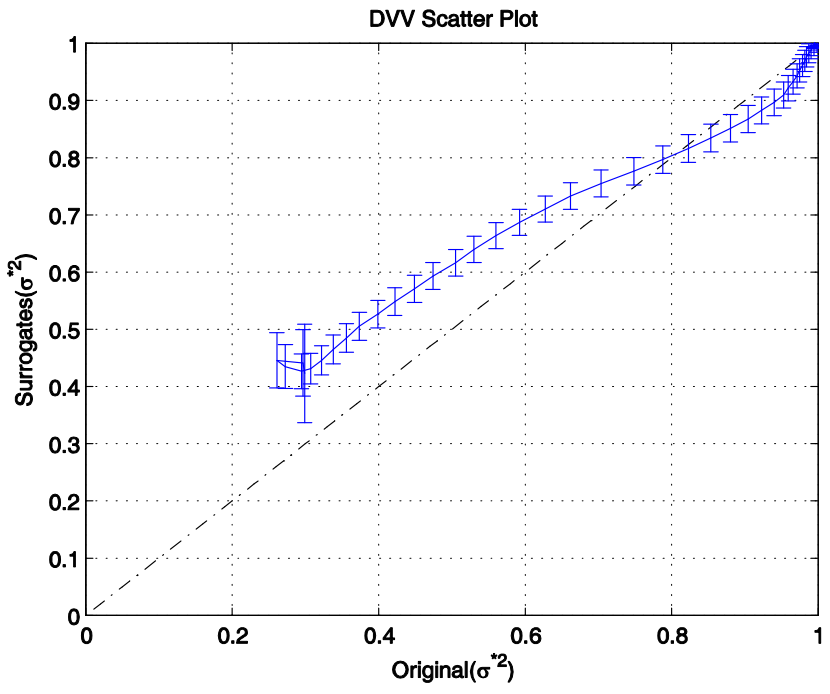

Fig.8. DVV scatter diagram

From Fig.8 it has been observed that the scattered plots are deviated from the bisector line and a high value 0.0678 of RMSE 
from Table. 2 is an indication of a deviation from the null hypothesis of linearity. Hence it can be said that the time series data are nonlinear in nature.

\section{CONCLUSION}

Traffic as observed in the study of the series of traffic volume is a complicated process. The current literature applies a variety of methodologies, ranging from time-frequency analysis, linear stochastic to parametric and nonlinear nonparametric analysis to predict its behavior. The present paper intends to characterize the statistical properties of long-term univariate traffic volume series regarding non-stationarity, determinism and nonlinearity. The analysis reveals a bi-modal distribution of the signal where the major mode is due to the working days of a week and the minor mode is due to the week-end low consumption of data. The observations from Smoothed Pseudo Wigner Ville Distribution (SPWVD) strongly recommends the time series data as Nonstationary. The DVV plot of the data ensures that the signal is relatively more stochastic in nature. The deviation in the DVV scatter plot indicates that the time series data are nonlinear in nature. This may also provide a good indication for adopting a variable prediction strategy according to the diversity of the statistical characteristics of traffic volume. In this paper, several characteristics regarding traffic volume's non-stationarity and nonlinearity are shown and not assumed to exist a priori. Even the proposed methodological framework is disengaged from assumptions regarding the statistical nature of the series under study. The proposed study of long-term traffic volume data forms an interesting yet not sufficiently developed field of research. Work in this area could give answers to prediction of the frequently observed boundary conditions caused by the phenomena of congestion and non-recurrent incidents and could provide a more sophisticated manner of selecting the proper longterm traffic, as well as detecting the time to shift between different prediction strategies.

\section{REFERENCES}

[1] S. Ansari, S.G. Rajeev and H.S. Chandrasekhar, "Packet Sniffing: Brief Introduction", IEEE Potentials, Vol. 21, No. 5, pp. 17-19, 2003.

[2] P. Asrodia and H. Patel, "Network Traffic Analysis using Packet Sniffer", International Journal of Engineering Research and Applications, Vol. 2, No. 3, pp. 854-856, 2012.

[3] M. Bhatia and S. Kaushal, "Online Statistical Internet Traffic Classification Approaches", International Journal of Advanced Research in Computer Science and Software Engineering, Vol. 2, No. 3, pp. 1-6, 2012.

[4] C. Partridge, D. Cousins, A.W. Jackson, R. Krishnan, T. Saxena and W.T. Strayer, "Using Signal Processing to Analyze Wireless Data Traffic", Proceedings of $1^{\text {st }}$ ACM workshop on Wireless Security, pp. 67-76, 2002.

[5] C. Barakat, P. Thiran, G. Iannaccon, C. Diot and P. Owezarski, "Modeling Internet Backbone Traffic at the Flow Level”, IEEE Transactions on Signal Processing, Vol. 51, No. 8, pp. 1-14, 2003.
[6] J. Timmer, "Power of Surrogate Data Testing with Respect to Nonstationary", Physical Review E, Vol. 58, No. 4, pp. 5153-5156, 1998

[7] Datamart, Available at: www.datamarket.com.

[8] R.G. Brown, R.F. Meyer and D.A. Desopo, "The Fundamental Theory of Exponential", Operations Research, Vol. 9, No. 5, pp. 673-687, 1961.

[9] E.S. Gardner, "Exponential Smoothing-The State of the ArtPart II", International Journal of Forecasting, Vol. 22, No. 4, pp. 637-666, 2006.

[10] J. Galtung, "Theory and methods of Social Research", British Journal for the Philosophy of Science, Vol. 4, No. 3, pp. 1-3, 1967.

[11] L. Cohen, "Time Frequency Distribution: A Review", Proceedings of the IEEE, Vol. 77, No. 7, pp. 941-981, 1989.

[12] E. Ebrahimzadeh, S.M. Alavi, B. Ahmad and A. Pakkhesal, "A Novel Approach for Detection of Deception using Smoothed Pseudo Wigner-Ville Distribution", Journal of Biomedical Science and Engineering, Vol. 6, No. 1, pp. 818, 2013.

[13] S.N.E. Pereira De, M. Custaud, J. Frutoso, I. Somody, C. Gharib and J. Fortrat, "Smoothed Pseudo Wigner-Ville Distribution as an Alternative to Fourier Transform in Rats", Autonomic Neuroscience: Basic and Clinical, Vol. 87, No. 2-3, pp. 258-267, 2001.

[14] A. Taebi and A.M. Hansen, "Analysis of Seismocardiographic Signals using Polynomial Chirplet Transform and Smoothed Pseudo Wigner-Ville Distribution", Electrical Engineering and Systems Science, pp. 1-6, 2017.

[15] Junhua Wu, Xinglin Chen and Zheshu Ma, "A Signal Decomposition Method for Ultrasonic Guided Wave Generated from Debonding Combining Smoothed Pseudo Wigner-Ville Distribution and Vold-Kalman Filter Order Tracking", Shock and Vibration, Vol. 2017, pp. 1-13, 2017.

[16] P. Flandrin, "Time-Frequency/Time-Scale Analysis", $1^{\text {st }}$ Edition, Academic Press, 1999.

[17] T.W. Anderson and D.A. Darling, "A Test of Goodness of Fit", Journal of the American Statistical Association, Vol. 49, pp. 765-769, 2014.

[18] T. Guatama, D.P. Mandic and M.M. Van Hulle, "The Delay Vector Variance Method for Detecting Determinism and Nonlinearity in Time Series", Physica D: Nonlinear Phenomena, Vol. 190, No. 3-4, pp. 167-176, 2004.

[19] R. Ray, M.H. Khodekar, K. Ghosh and A.K. Bhattacharjee, "Scaling and Nonlinear Behaviour of Daily mean Temperature Time Series across India", Chaos, Solitons and Fractals, Vol. 84, pp. 9-14, 2016.

[20] T. Schreiber and A. Schmitz, "Improved surrogate data for nonlinearity tests", Physical Review Letters, Vol. 77, pp. 635-638, 1996.

[21] P. Mansier, J. Clairambault, N. Charlotte, C. Medique, C. Vermeiren, G. LePape, F. Carre, A. Gounaropoulou and B. Swynghedauw, "Linear and Non-Linear Analyses of Heart Rate Variability: a Minireview", Cardiovascular Research, Vol. 31, No. 3, pp. 371-379, 1996.

[22] T. Schreiber and A. Schmitz, "Surrogate Time Series", Physica D: Nonlinear Phenomena, Vol. 142, No. 3-4, pp. 346-382, 2000. 
[23] T. Gautama, D. Mandic and M.V. Hulle, “A Differential Entropy based method for Determining the Optimal Embedding Parameters of a Signal", Proceedings of IEEE International Conference on Acoustics, Speech, and Signal Processing, pp. 113-117, 2003.

[24] Y. Yuan, Y. Li and D.P. Mandic, "Comparison Analysis of Embedding Dimension between Normal and Epileptic EEG Time Series", Journal of Physiological Sciences, Vol. 58, No. 4, pp. 239-247, 2008.

[25] J. Theiler, S. Eubank, A. Longtin and B. Galdrikian, "Testing for Non Linearity in Time Series:the Method of Surrogate Data", Physica D: Nonlinear Phenomena, Vol. 58, No. 1-4, pp. 77-94, 1992.
[26] H.V. Ravinder, "Determining The Optimal Values of Exponential Smoothing Constants-does Solver Really Work?", American Journal of Business Education, Vol. 6, No. 3, pp. 347-360, 2013.

[27] S. Ata, M. Murata and H. Miyahara, "Analysis of Network Traffic and its Application to Design of High-Speed Routers", IEICE Transactions on Information and Systems, Vol. 83, No. 5, pp. 988-995, 2000.

[28] R. Ray, M.H. Khondekar, K. Ghosh and A.K. Bhattacharjee, "Memory Persistency and Non Linearity in Daily Mean Dew Point across India", Theoretical and Applied Climatology, Vol. 124, No. 1-2, pp. 119-128, 2015. 